Reprod. Nutr. Dévelop., 1980, 20 (4 B), 1197-1202.

\title{
Obtention des digesta parvenant au gros intestin par fistulation iléo-colique post-valvulaire : Note préliminaire
}

\author{
par Béałrice DARCY, J. P. LAPLACE, P. A. VILLIERS \\ Laboratoire de Physiologie de la Nutrition, I.N.R.A., \\ 78350 jouy en Josas, France
}

Summary. Collection of digesta flowing into the large intestine after post-ileocolic valve fistulation : Preliminary results.

The various techniques of ileoileal of ileocaecal reentrant fistulation now used to collect digesta flowing from the small into the large intestine, do not provide an accurate estimate of digestion in the small intestine. Their main disadvantages are : an erroneous description of the kinetics of food passage (collection proximal to the valve or bypass of the ileocaecal valve) and changes in the ileal microflora.

A new surgical technique is described (fig. 1) for collecting the digesta as they have been propelled through the ileocaecal valve, thus maintaining the functional role of the valve. When using such a technique, the digesta collected into a rubber bag must be returned to the proximal colon by the investigator. Using this post-ileocolic valve fistulation technique, the disorders commonly reported with the other techniques are avoided, and a true picture of food passage at the ileocaecal valve is obtained.

La fistulation réentrante de l'intestin grêle permet la collecte totale des digesta et leur échantillonnage, et par là l'établissement de la cinétique de passage des divers matériaux alimentaires. Sa mise en œuvre permet donc de dresser un bilan de la digestion au sife concerné. L'intérêt potentiel de cette méthodologie a conduit divers auteurs à utiliser la fistulation réentrante de l'iléon terminal pour évaluer la digestion dans l'intestin grêle (Holmes, Bayley et Horney, 1973 ; Horszczaruk et Zebrowska, 1973 ; Braude, Fulford et Low, 1976 ; Drochner ef Hazem, 1976 ; Low, Partridge et Sambrook, 1978). Pourtant, la fistulation réentrante iléo-iléale pose dans le cas de l'iléon du Porc des problèmes considérables (Laplace et Borgida, 1976), de type obstruction, systématiquement rencontrés par tous les auteurs cités précédemment. Aucune des tentatives de solution jusqu'ici mises en œuvre, concernant soit la technologie des canules, soit l'alimentation post-opératoire des porcs, n'a permis d'éviter les désordres habituels chez les animaux ainsi préparés.

On peut reprocher à la fistulation iléo-iléale, dans la perspective d'un bilan de la digestion dans l'infestin grêle, de conduire à une dérivation prématurée des digesta donc de fausser leur cinétique apparente de passage. Les difficultés mentionnées plus ou moins explicitement par tous les auteurs ont conduit certains à proposer une fistulation iléo-cæcale (Easter et Tanksley, 1973 ; Hazem et Drochner, 1976). Mais cette autre solution n'exclut pas totalement le risque d'obstruction. De plus, elle rend dou- 
teuse toute cinétique en raison de la mise hors circuit de la jonction iléo-cæcocolique dont le rôle exact reste à ce jour mal précisé. Elle rend en outre suspecte toute information qualitative du fait de l'envahissement de l'iléon par une flore de type cæcocolique. Ces divers inconvénients pourraient constituer une source d'erreur fort importante dans l'évaluation de la composition en acides aminés, qui n'a été établie jusqu'ici que chez des porcs à fistulation iléo-iléale (Zebrowska, Buraczewska et Buraczewski, 1978 ; Low, 1979). Enfin les 2 types de fistulation (iléo-iléale ou iléocæcale) impliquent comme toute fistulation réentrante une transsection intestinale en elle-même perturbatrice (Laplace, 1979). Ce dernier inconvénient peut cependant être évité par l'usage de la canule décrite par Ivan (1974).

Dans ce contexte, nous nous sommes attachés à développer une technique (1) excluant la transsection intestinale mais aussi (2) préservant le rôle fonctionnel de la jonction iléo-cæco-colique, et (3) évitant la dérivation prématurée des digesta. Par ailleurs, nous avons retenu le choix de canules d'un diamètre supérieur à ce qui a été jusqu'ici utilisé, et d'un dispositif réduisant au minimum la longueur de tube inerte à franchir, au besoin en excluant la possibilité d'un pontage des canules, donc en exigeant l'intervention expérimentale pour la réintroduction.

\section{Matériel et méthodes.}

Dix porcs d'un poids vif compris entre 60 et $75 \mathrm{~kg}$ ont été utilisés pour la mise au point progressive de la forme de canule la plus adaptée, de la technique opératoire
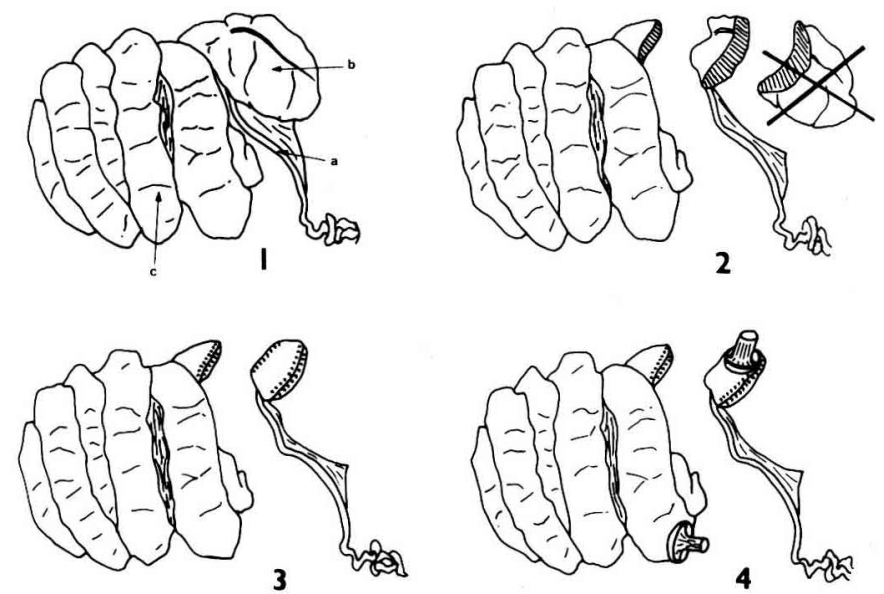

FIG. 1. - Schéma de principe des diffèrentes étapes de réalisation de la fisfulation iléo-colique post-valvulaire.

1. - Carrefour iléo-caeco-colique intact ( $a=$ iléon, $b=$ caecum, $c=$ côlon hélicoïdal).

2. - Isolement du segment caeco-colique comportant l'abouchement de l'iléon, ef résection du crecum.

3. - Fermeture de l'about proximal du côlon hélicoïdal et construction de la poche de tissu cæcocolique enfermant la saillie valvulaire.

4. - Mise en place d'une canule de dérivation dans la poche cæco-colique et d'une canule pour la réintroduction extemporanée des digesta dans le côlon. 
et du dispositif de collecte des contenus. En définitive, le principe de l'opération schématisé dans la figure 1 , consiste à isoler entre deux sections la jonction cæco-colique. Le cæcum est réséqué et l'about proximal du côlon est refermé. Le segment cæcocolique, dans lequel émerge la saillie de la valvule iléo-cæcale, est utilisé pour la construction d'une petiłe poche sphérique montée sur une canule dont l'embase coiffe la saillie valvulaire. On utilise, pour cela, une canule en verre (Livingstone ef al., 1977) de diamètre intérieur $19 \mathrm{~mm}$ (extérieur $22 \mathrm{~mm}$ ) et dont l'embase est en forme de cloche (intérieur $33 \mathrm{~mm}$ ). Ce montage, dénommé fistulation iléo-colique post-valvulaire, permet l'éjection des digesta par la valvule directement dans le tube de la canule. Leur collecte est assurée dans un ballon fixé sur le flanc de l'animal. Parallèlement, une canule simple (silicone médical ; diamètres : intérieur $16 \mathrm{~mm}$, extérieur $22 \mathrm{~mm}$ ) montée dans la première spire du côlon hélicoïdal permet la restitution par l'expérimentateur des contenus ainsi collectés.

Les trois derniers porcs préparés comme indiqué ci-dessus ont en outre été équipés d'électrodes chroniques intra-pariétales souples (Laplace, 1978) au niveau de l'iléon, pour une première évaluation des éventuelles conséquences d'une telle préparation sur les activités électromyographiques de l'intestin grêle. Les enregistrements ont été réalisés pendant des périodes de $7 \mathrm{~h}$ après le repas, et dans 3 cas pendant des périodes de $24 \mathrm{~h}$.

A titre comparatif, un porc a été équipé d'une canule en T (réalisée en verre et de même diamètre que dans le cas précédent) sur l'iléon terminal, et d'une canule de réintroduction sur le cæcum. Comme dans le cas précédent, et à la différence des montages réentrants iléo-cæcaux, la collecte est réalisée dans un ballon externe, et la restitution des digesta assurée par l'expérimentateur.

\section{Résultats et discussion.}

La technique proposée pour dériver les digesta lors de leur émission par la valvule iléo-cæcale permet de répondre à l'ensemble des critiques formulées précédemment. Elle excluł toute atteinte chirurgicale de l'iléon et préserve le rôle fonctionnel de la jonction iléo-colique. Elle permeł donc de collecter les digesta tels qu'ils sont réellement émis vers le gros intestin. Les phénomènes classiques d'obstruction progressive de l'iléon n'ont pas été observés avec cette préparation.

Ce montage post-valvulaire permet des collectes dans de bonnes conditions durant environ 3 semaines. Mais le recul progressif de la canule de verre, ébranlée par les mouvements de l'animal et entraînée par le poids du ballon de collecte, conduit à un certain accroissement du volume de la poche cæco-colique. Dès lors les digesta émis tendent à s'y accumuler au lieu d'être éjectés directement dans la canule. Ce processus est occasionnellement responsable d'obstructions de la canule de dérivation. Néanmoins ce problème tardif reste sans commune mesure avec les difficultés rencontrées dans le cas des préparations classiques. Le seul inconvénient véritable du montage proposé réside dans la nécessité de l'intervention de l'expérimentateur pour assurer la restitution des digesta dans le côlon. Cet aspect devra faire l'objet d'une analyse particulière notamment en ce qui concerne les modalités de la réintroduction. 
Les premières observations réalisées, lors d'ingestion à $9 \mathrm{~h}$ d'un régime semipurifié à base d'amidon de maïs (Cuber et Laplace, 1979) semblent montrer que par rapport à la cinétique observée au niveau de l'iléon terminal (canule en $\mathrm{T}$ ), les digesta sont recueillis environ $1 \mathrm{~h}$ plus tard lors de dérivation post-valvulaire. Cependant, le nombre très restreint de données comparatives disponibles ne permet pas encore d'établir les cinétiques caractéristiques des 2 préparations. Cela étant, chez les porcs préparés par fistulation iléo-colique post-valvulaire, les volumes de digesta collectés en $7 \mathrm{~h}$ après un repas à $9 \mathrm{~h}$ varient assez largement d'un porc à l'autre. Ainsi, pour des repas de $800 \mathrm{~g}$ d'un aliment semi-purifié (farine diluée par 2 fois son poids d'eau), on collecte en $7 \mathrm{~h}$ environ $710 \mathrm{ml}$ de digesta chez un porc contre $320 \mathrm{ml}$ seulement chez un autre animal. La variation liée au niveau d'ingestion est manifeste avec par exemple des volumes recueillis de l'ordre de 260,320 et $390 \mathrm{ml}$ respectivement pour des repas de 600,800 et $1000 \mathrm{~g}$ du même aliment semi-purifié. L'ingestion d'un aliment standard (céréales, soja ; dilution par 2 fois son poids d'eau) conduit à collecter en $7 \mathrm{~h}$ des volumes nettement plus importants, de l'ordre de 630 et $850 \mathrm{ml}$ pour des
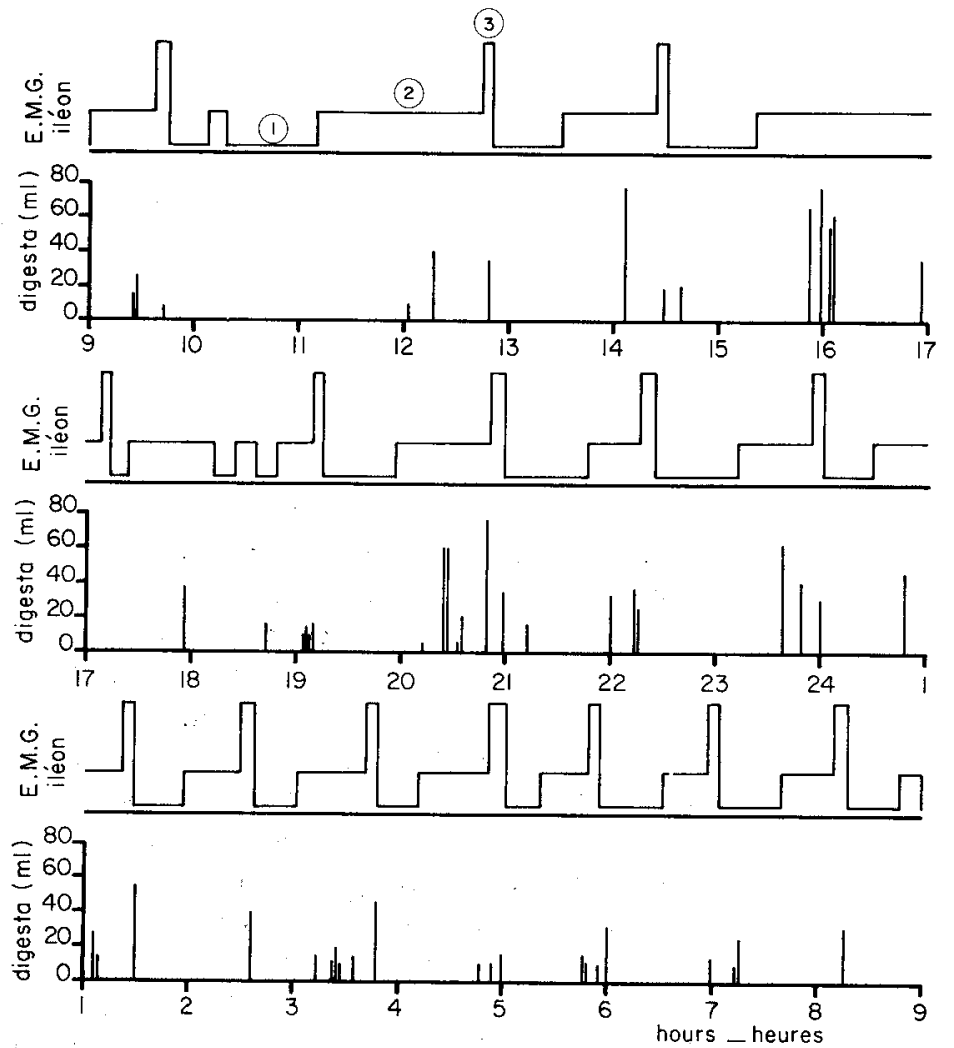

FIG. 2. - Représentation graphique de la succession chronologique des complexes myoélectriques (phases 1-2-3) au niveau de l'iléon (électro-myogramme-E. M. G.-dérivé à $12 \mathrm{~cm}$ en amont de la jonction iléo-cæco-colique) et des émissions de digesta au niveau de l'ostium iléo-cæco-colique, pendant une période de $24 \mathrm{~h}$ chez un porc recevant 2 repas quotidiens, d $9 \mathrm{~h}$ et $16 \mathrm{~h}$, de $1000 \mathrm{~g}$ chacun (aliment semi-purifié). 
repas de 500 et $700 \mathrm{~g}$ contre 560 ef $710 \mathrm{ml}$ chez le même porc ingérant 600 ou $800 \mathrm{~g}$ de l'aliment semi-purifié. Enfin, une dilution accrue de l'aliment standard conduit également à une augmentation du volume de digesta franchissant la jonction iléo-crecocolique : pour un même ingéré de $700 \mathrm{~g}$, le volume collecté passe de $850 \mathrm{ml}$ lorsque l'aliment est dilué par 2 fois son poids à $1200 \mathrm{ml}$ lorsqu'il est dilué par 3 fois son poids d'eau. Il semblerait donc que les variations quantitatives de l'évacuation gastrique, liées au niveau d'ingestion, à la composition ou à la dilution de l'aliment, se répercutent sur le débit des digesta au niveau de la jonction iléo-cæco-colique.

Si satisfaisante que la fistulation iléo-colique postvalvulaire puisse paraître au plan théorique, il n'est pas exclu que la dissection chirurgicale du carrefour iléocæco-colique conduise à léser l'innervation de cette région. Cependant, l'observation directe à travers la canule de l'émission des digesta montre la persistance d'une véritable contractilité de la saillie valvulaire. Les mouvements de cette dernière, en continuité avec l'iléon terminal, semblent traduire l'aboutissement de la propagation d'ondes péristaltiques poussant les bols de digesta émis au niveau de l'ostium iléocæco-colique. De fait, les enregistrements électromyographiques réalisés montrent dans tous les cas la persistance d'une organisation normale des électromyogrammes en complexes myoélectriques (MMC) sur l'iléon terminal (fig. 2). Au cours des $72 \mathrm{~h}$ d'enregistrements continus, on dénombre en moyenne $14 \mathrm{MMC}$ par $24 \mathrm{~h}$; la durée des phases qui les constituent est respectivement de 53,3 \pm 3,7 $\mathrm{mn}$ (phase l, quiescence), de 44,9 $\pm 5,5 \mathrm{mn}$ (phase II, activité de pointes irrégulière) et de 4 à $6 \mathrm{mn}$ (phase III, activité de pointes régulière), valeurs normales au niveau de l'iléon distal lors d'ingestion d'un régime semi purifié hautement digestible. Enfin, l'enregistrement simultané de chacune des émissions de digesta au niveau de l'ostium iléo-cæc:colique (fig. 2) permet de constater que la propulsion des bols de contenus s'effectue bien pour l'essentiel au cours des périodes d'activité irrégulière comme il a été antérieurement décrit (Laplace ef Roman, 1979). L'éjection vers le gros intestin des derniers bols d'une série chronologique correspondant à une phase d'activité irrégulière survient en général alors que l'activité régulière immédiatement consécutive envahit les $30 \mathrm{~cm}$ les plus distaux de l'iléon. Au total, la fistulation iléo-colique postvalvulaire ne semble donc pas perturber les activités motrices de l'intestin grêle.

En conclusion : Ce travail préliminaire montre qu'il est possible d'obtenir chez le Porc des digesta tels qu'ils sont normalement transférés de l'intestin grêle vers le gros intestin, en préservant l'intégrité fonctionnelle de l'intestin grêle.

Journées Ingestion-Digestion-Absorption de l'Association française de Nutrition. Paris, 15-16 novembre 1979.

\section{Références}

BRAUDE R., FULFORD R. J., LOW A. G., 1976. Studies on digestion and absorption in the intestines of growing pigs. Measurements of the flow of digesta and $\mathrm{pH}$. Br. J. Nutr., 36, 497-510.

CUBER J. C., LAPLACE J. P., 1979. Evacuation gastrique de la matière sèche d'un régime semi-purifié à base d'amidon de maïs chez le porc. Ann. Biol. anim. Bioch. Biophys., 19, 899-905.

DROCHNER W., HAZEM A. S. von, 1976. Entwicklung einer Umleitungskanülentechnik für das jleum beim Schwein, Z. Tierphysiol. Tierernöhr. Futfermittelkde, 37, 26-30. 
EASTER R. A., TANKSLEY T. D., 1973. A fechnique for reentrant ileo-caecal cannulation of swine. J. Anim. Sci., 36, 1099-1103.

HAZEM A. S. von, DROCHNER W., 1976. Extracorporaler ileocaecaler Bypass unter Umgehung des Ostium ileocaecale beim Schwein. Z. Versuchstierk., 18, 303-306.

HOLMES J. H. G., BAYLEY H. S., HORNEY F. D., 1979. Digestion and absorption of dry and high moisture maize diets in the small and large intestine of the pig. Br. J. Nutr., 30, 401-410.

HORSZCZARUK F., ZEBROWSKA T., 1973. Permanent intestinal fistulae for the study of digestion in pigs. 3) Establishment of reentrant fistulae of the small intestine (Polish). Roczn. Nauk. Roln., 95, 157-168.

IVAN M., 1974. A new type of reentrant cannula designed for use in the small intestine of the pig. Austr. Vet. J., 50, 547-552.

LAPLACE J. P., 1978. Organisation de la motricité de l'intestin grêle chez le porc et influence de l'alimentation. Ann. Zootech., 27, 377-408.

LAPLACE J.P., 1979. Stomach and small intestine motility in the Pig. Electromyography in nutritional studies. In LOW A. G., PARTRIDGE I. G., Current concepts of digestion and absorption in pigs, 1 Vol., N. I. R. D., Reading (UK) (In Press).

LAPLACE J. P., BORGIDA L. P., 1976. Problèmes physiologiques posés par la fistulation réentrante chronique de l'iléon chez le Porc. Etude bibliographique et expérimentale. Ann. Zoofech., 25, 361-371.

LAPLACE J. P., ROMAN C., 1979. Activités de la musculature gastro-intestinale ef mourements des contenus digestifs. Ann. Biol. anim. Bioch. Biophys., 19, 849-879.

LIVINGSTONE R. M., FOWLER V. R., WHITE F., WENHAM G., 1977. Annealed glass cannulae for use in digestion studies with pigs. Vet. Rec., 101, 368.

LOW A. G., 1979. Studies on digestion and absorption in the intestines of growing pigs. 6) Measurements of the flow of amino-acids. Br. J. Nutr., 41, 147-156.

LOW A. G., PARTRIDGE I. G., SAMBROOK I. E., 1978. Studies on digestion and absorption in the intestines of growing pigs. 2) Measurements of the flow of dry matter, ash and water. Br. J. Nutr., 39, 515-526.

ZEBROWSKA T., BURACZEWSKA L., BURACZEWSKI S., 1978. The apparent digestibility of aminoacids in the small infestine and in the whole digestive tract of pigs fed diets containing different sources of protein. Roczn. Nauk. Roln. Ser. B, 99, 87-98. 